\title{
Evaluation of health parameters, use of drugs, and alcohol intake among an elderly population in São José dos Campos, SP, Brazil
}

\author{
Simone Bernardes de Paula1, Patricia Fernanda Braga Mendonça', Mateus Bertolini Fernandes dos Santos², \\ Jarbas Francisco Fernades dos Santos ${ }^{1,3}$, Leonardo Marchini ${ }^{4}$
}

\author{
${ }^{1}$ Universidade do Vale do Paraiba - Univap, School of Dentistry, Area of Prosthodontics, São José dos Campos, SP, Brasil \\ ¿Universidade Estadual de Campinas - Unicamp, Piracicaba Dental School, Department of Prosthodontics and Periodontics, Piracicaba, SP, Brasil \\ 3Universidade de Taubaté - UNITAU, Department of Dentistry, Area of Prosthodontics, Taubaté, SP, Brasil \\ ${ }^{4}$ University of lowa, College of Dentistry, Department of Preventive and Community Dentistry, lowa City, IA, USA
}

\begin{abstract}
Aim: To evaluate health parameters (blood pressure, heart rate and blood glucose), the use of medicines and alcohol consumption in elderly residents in the city of São José dos Campos, SP, Brazil. Methods: A sample of 500 elderly individuals (98 men and 402 women, with mean age of 69.5 years) was examined. Personal data and medicines used by the patients were recorded, general health aspects were assessed and the Alcohol Use Disorders Identification Test (AUDIT) was applied to survey alcohol consumption. Results: Two hundred and four patients $(40.8 \%)$ presented high blood pressure and 93 patients (18.6\%) had hyperglycemia. The most used pharmacological groups were antihypertensive drugs, antilipidemics, drugs to control hypothyroidism and hypoglycemic agents. Possible pharmacological interactions with alcohol were present in $60.9 \%$ of the used medicines. The AUDIT results showed that $91 \%$ of the sample presented a low-level intake of alcohol and only $1 \%$ presented characteristics of alcohol addiction. Associations were found among AUDIT scores with age $(p=0.037)$, since a larger number of alcohol addicts were found among younger patients, and use of drugs ( $p=0.046)$, since patients who consumed more daily medicines made less use of alcohol (low-level). Conclusions: The studied sample presented a high prevalence of hypertension and relatively low incidence of diabetes. A low use of drugs was verified and women made more use of daily medications than men. Low alcohol consumption was also observed, and women consumed less alcohol than men.
\end{abstract}

Keywords: medicines; drugs; alcohol; elderly; general health.

\section{Introduction}

Received for publication: February 04, 2014 Accepted: March 24, 2014

Correspondence to: Mateus Bertolini Fernandes dos Santos Departamento de Prótese e Periodontia Faculdade de Odontologia de Piracicaba - UNICAMP

Av. Limeira, 901 - CEP 13414-903 Piracicaba, SP, Brazil

Phone: +55 19 2106-5297

E-mail: mateusbertolini@yahoo.com.br
The whole world is experiencing a growth in the elderly population. A demographic study in Brazil showed that in 10 years (1998-2008) the elderly increased from $8.8 \%$ to $11.1 \%$ of the entire population ${ }^{1}$, totalizing approximately 21 million people, which is more than the number of elderly in the United Kingdom, France, and Italy.

Alcohol abuse among the elderly has been called an "invisible epidemic" because it often does not follow stereotypes and therefore goes unnoticed. As the elderly population continues to increase, there is a growing need to re-evaluate the problem of alcoholism in this group of the population ${ }^{2}$. Abusive intake of 
alcohol by the elderly can have serious health consequences, undermining the length and quality of their lives ${ }^{3}$. Alcohol addiction prevalence is currently lower in people over 65 years compared to other age groups, but there is some evidence of a growing proportion of elderly people who use alcohol at inappropriate levels. Because older people are more vulnerable to alcohol's effects, the current definitions of abuse and alcohol dependence may also be more stringent for this population ${ }^{4}$.

Although alcohol-related problems are common among the elderly, physicians almost never recognize them early because of the difficulty in making early diagnoses, since the problems related to alcohol are not usually addressed in elderly patients 5 , especially in women ${ }^{6}$. However, the elderly are particularly prone to the adverse consequences of alcohol use, such as depression, sleep disorders, gastritis, hypertension, problems with heart rate, diabetes, falls, bradycardia and arrhythmias. This is due to functional decreases caused by age-related physiological changes in the distribution of alcohol, the effects of alcohol on the central nervous system and the increased use of medications associated with age $^{7-8}$.

Furthermore, the concomitant use of drugs and alcohol in the elderly may decrease the effectiveness of medications, increase the incidence of undesirable drug side effects ${ }^{7,9}$, and also affect negatively the elderly's general health. In view of this, the aim of this study was to evaluate some health parameters (blood pressure, heart rate and blood glucose), the use of medicines, and alcohol use in an elderly sample of residents of the city of São José dos Campos, SP, Brazil. The tested hypothesis was that the use of drugs among this population is low ${ }^{10}$, but an important impact on general health parameters might be found among alcohol abusers.

\section{Material and methods}

\section{Participants}

Considering a total elderly population of 40,524 individuals in São José dos Campos, a sample of 500 patients was included in the study, which has $86.64 \%$ power to represent the target population (Minitab 15 software package). The individuals were recruited among the community centers for the independent elderly registered in the Municipal Committee for the Elderly, until the desired sample size was achieved. The participants signed an informed consent form approved by the University of Vale do Paraíba Ethics Committee (Protocol \#H105/CEP/2010).

\section{Evaluation of personal data, general health, and drugs administration}

Personal data of the patients, such as name, age, sex, and education level (divided into illiterate, primary school, high school, college and post-graduation, complete or incomplete) were registered during initial interviews. For general health evaluation, blood pressure and glucose were checked; $140 \mathrm{mg} / \mathrm{dL}$ was considered the normal value of postprandial blood glucose, according to the American Diabetes Association ${ }^{11}$, and $120 / 80 \mathrm{mmHg}$ the normal value for blood pressure. A single professional made all examinations following a standard procedure. The examinations and interviews take place at the community center where the participants were recruited.

Drugs used by the patients were also recorded, and the names, frequency of intake and possible pharmacological interactions with alcohol were evaluated.

\section{Alcohol intake evaluation}

For such evaluation, the Alcohol Use Disorders Identification Test (AUDIT) was applied to the patients. This test was designed by the World Health Organization to identify disorders caused by alcohol abuse in facilities for primary health care ${ }^{4}$, and it has been shown to be valid in the detection of dangerous alcohol use in large-scale studies with active adults and elderly patients ${ }^{12-13}$. A BrazilianPortuguese version of the test had been previously validated $^{10,14}$ and was used in this study.

All interviews were conducted by a single operator, who had been previously calibrated for interviewing and completing the questionnaire forms.

\section{Data analysis}

The collected data were tabulated and descriptive analysis was made. Parametric tests were used due to normal distribution of the data (Kolmogorov-Smirnov test). One-way ANOVA was used for intergroup comparisons and the chisquared test was used for verification of possible associations. The significance level was set at $5 \%$

Chart 1 - Possible interactions of different pharmacological groups with alcohol.

\begin{tabular}{ll}
\hline Pharmacological groups & Possible interactions with alcohol \\
Antibiotics - Metronidazol & Vomit, palpitations, low blood pressure, breathing difficulty \\
Antibiotics - Eritromicine & Liver damage, vomit, palpitations, low blood pressure, breathing difficulty, inhibits the effect of the drug \\
Anticonvulsants & Breathing difficulty, inhibits the effect of the drug, dizziness, intoxication \\
Antidepressives & Inhibits the effect of the drug, increases the sedative effect \\
Antifungals & Liver damage \\
Antihypertensives & Dizziness, fainting, cardiac arrhythmia \\
Antiarrhythmics & Further depresses normal heart function \\
Anxiolytics I & nhibits the effect of the drug, increases the sedative effect, risk of coma \\
Cholesterol control drugs & Liver damage, stomach bleeding \\
Insulin & Vomit, palpitations, headache, hypoglycemia \\
\hline
\end{tabular}




\section{Results}

The sample comprised 98 men and 402 women and the mean age was 69.5 years old, with 101 and 60 being the highest and lowest ages, respectively. As much as $54.2 \%$ of the participants had only primary education, $13.6 \%$ completed college, $1.2 \%$ had post-graduation education and $2.4 \%$ were illiterate.

Two hundred and four individuals $(40.8 \%)$ presented high blood pressure and $39(7.8 \%)$ hypotension. The mean rate of the patients was 71.4 , with 100 and 43 being the highest and lowest values, respectively. With regard to blood glucose, 93 patients $(18.6 \%)$ presented hyperglycemia and $6(1.2 \%)$ presented hypoglycemia.

Regarding the use of drugs, the most used pharmacological groups were antihypertensives, antilipidemics, hypothyroidism control drugs, and hypoglycemic agents (Table 1). The mean daily drug use was 2.2 and the highest value was 14 drugs taken on a daily basis. The most used drug in this sample was simvastatin (antilipidemic), followed by captopril (antihypertensive). Among all the drugs used by the patients, $60.9 \%$ presented possible pharmacological interactions with alcohol. When sex was compared regarding the use of drugs, it was found

Table 1 - Distribution of the most used pharmacological groups.

\begin{tabular}{lll}
\hline Pharmacological group & $\mathbf{n}$ & $\%$ \\
Antihypertensives & 416 & $37.48 \%$ \\
Antilipidemics & 106 & $9.54 \%$ \\
Hypothyroidism control drugs & 86 & $7.75 \%$ \\
Hypoglycemiant drugs & 67 & $6.04 \%$ \\
Osteoporosis control drugs & 57 & $5.14 \%$ \\
Anticoagulants & 50 & $4.50 \%$ \\
Anxiolytics & 49 & $4.41 \%$ \\
Antiulcerous & 40 & $3.60 \%$ \\
Antiarrhythmic & 19 & $1.71 \%$ \\
Hypoglycemia control drugs & 18 & $1.62 \%$ \\
\hline
\end{tabular}

that women consumed more drugs than men $(p=0.003)$.

Table 1 presents the possible interactions of different pharmacological groups used by the patients in this study and the possible interactions with alcohol.

When different blood pressure groups were compared by age, blood glucose levels, heart rate levels and number of drugs used daily, it was observed that patients presenting hypertension also presented higher heart rate $(p=0.015)$. No significant differences were found among different blood pressure groups to the other variables.

The AUDIT results showed that $91 \%$ of the interviewed elders present a low-level intake of alcohol and only $1 \%$ presented characteristics of alcohol addiction. Table 2 shows the comparisons of different AUDIT groups regarding age, blood glucose levels, heart rate levels and number of daily used drugs. Significant differences among different AUDIT groups regarding the age $(p=0.037)$ were found. A large number of alcohol addicts were found among younger patients and use of drugs $(p=0.046)$, the patients who consumed more medicines daily were those who consumed less alcohol.

Table 3 presents the associations among sexes and blood pressure, blood glucose, educational level, and AUDIT classifications. An association between sex and education level $(p=0.017)$ was verified, where women predominated among those who had elementary school education and men, among those who with high school education. Another association was found between sex and AUDIT classification $(p<0.001)$, where women presented lower scores of alcohol intake than men.

No correlation was found between blood pressure and glucose and the AUDIT scores.

\section{Discussion}

In this study, a very marked predominance of women $(80.4 \%)$ was observed. The elderly subjects had a mean age of 69.5 years, with a minimum of 60 and a maximum of 101 years. These results were similar to those described by De Deco et al. ${ }^{9}$, who reported a mean age of 74.3 years and

Table 2 - Comparison of age, blood glucose, heart rate and use of drugs among AUDIT groups.

\begin{tabular}{|c|c|c|c|c|c|c|}
\hline AUDIT & & Average & $\begin{array}{l}\text { Standard } \\
\text { Deviation }\end{array}$ & $\mathrm{n}$ & $\begin{array}{c}\text { Confidence } \\
\text { interval }\end{array}$ & $p$-value \\
\hline \multirow[t]{3}{*}{ Age } & Low risk & 69.6 & 6.6 & 455 & 0.6 & $0.037^{*}$ \\
\hline & Addiction & 62.6 & 2.4 & 5 & 2.1 & \\
\hline & Harmful abuse & 68.5 & 6.3 & 39 & 2.0 & \\
\hline \multirow[t]{3}{*}{ Blood glucose } & Low risk & 120.5 & 35.7 & 455 & 3.3 & 0.534 \\
\hline & Addiction & 133.2 & 46.4 & 5 & 40.7 & \\
\hline & Harmful abuse & 116.1 & 22.1 & 39 & 6.9 & \\
\hline \multirow[t]{3}{*}{ Heart rate } & Low risk & 71.3 & 8.2 & 455 & 0.8 & 0.143 \\
\hline & Addiction & 65.8 & 4.6 & 5 & 4.0 & \\
\hline & Harmful abuse & 73.0 & 7.3 & 39 & 2.3 & \\
\hline \multirow[t]{3}{*}{ Use of drugs } & Low risk & 2.3 & 1.9 & 455 & 0.2 & $0.046^{*}$ \\
\hline & Addiction & 1.6 & 1.7 & 5 & 1.5 & \\
\hline & Harmful abuse & 1.5 & 1.4 & 39 & 0.5 & \\
\hline
\end{tabular}

The asterisk $\left(^{*}\right)$ indicates a statistically significant difference (One-way ANOVA). 
Table 3 - Association of sexes and blood pressure, blood glucose, educational level and AUDIT groups.

\begin{tabular}{lllllllll}
\hline & & \multicolumn{2}{c}{ Women } & \multicolumn{2}{c}{ Men } & \multicolumn{2}{c}{ Total } & \multicolumn{1}{c}{$\boldsymbol{p}$-value } \\
Blood pressure & Hypotension & 35 & $9 \%$ & 4 & $4 \%$ & 39 & $8 \%$ & 0.293 \\
& Hypertension & 161 & $40 \%$ & 43 & $44 \%$ & 204 & $41 \%$ & \\
& Normal & 206 & $51 \%$ & 51 & $52 \%$ & 257 & $51 \%$ & \\
\hline Blood glucose & High & 75 & $19 \%$ & 18 & $18 \%$ & 93 & $19 \%$ & 0.694 \\
& Low & 4 & $1 \%$ & 2 & $2 \%$ & 6 & $1 \%$ & \\
& Normal & 323 & $80 \%$ & 78 & $80 \%$ & 401 & $80 \%$ & \\
\hline Education level & Illiterate & 11 & $3 \%$ & 1 & $1 \%$ & 12 & $2 \%$ & $0.017^{*}$ \\
& Primary School & 231 & $57 \%$ & 40 & $41 \%$ & 271 & $54 \%$ & \\
& High School & 107 & $27 \%$ & 36 & $37 \%$ & 143 & $29 \%$ & \\
& College & 48 & $12 \%$ & 20 & $20 \%$ & 68 & $14 \%$ & \\
& Post-graduation & 5 & $1 \%$ & 1 & $1 \%$ & 6 & $1 \%$ & \\
\hline AUDIT & Low risk & 374 & $93 \%$ & 81 & $83 \%$ & 455 & $91 \%$ & $<0.001^{*}$ \\
& Addiction & 1 & $0 \%$ & 4 & $4 \%$ & 5 & $1 \%$ & \\
\hline Total & Harmful abuse & 26 & $6 \%$ & 13 & $13 \%$ & 39 & $8 \%$ & \\
\hline
\end{tabular}

The asterisk $\left(^{*}\right)$ indicates a statistically significant association (Chi-square test).

women also accounted for the vast majority, $70.3 \%$ of the sample.

The American Heart Association (AHA) considers as normal blood pressure when systolic/diastolic pressure is 120 / $80 \mathrm{mmHg}$ and persons presenting blood pressure of 140/90 $\mathrm{mmHg}$ are considered to be hypertensive, although one may argue about the fact that elderly persons may have different parameters in blood pressure than the AHA recommends. However, the Blood Pressure UK, formerly known as Blood Pressure Association, suggests that the level for high blood pressure does not change with age ${ }^{15}$. Based on these parameters, in the studied sample, $51.4 \%$ of the elders presented normal blood pressure values, $40.8 \%$ were hypertensive, and $7.8 \%$ presented hypotension. In Campinas, SP, Brazil, Zeitune et al. ${ }^{16}$ found a hypertension prevalence of $51.8 \%$ among 426 adults aged 60 years or older, with higher incidence in women (55.9\%). In Japan, blood pressure was measured in 499 elderly community dwellers, with a mean age of 80 years, and mean systolic and diastolic blood pressures were $149.6 \mathrm{mmHg}$ and $78.5 \mathrm{mmHg}$ respectively ${ }^{17}$. De Deco et al. ${ }^{9}$ showed a slightly higher systolic pressure in the group of institutionalized elders compared with community dwellers, and diastolic blood pressure was higher in the community dwellers. Hypertension is considered an aggravating factor in health and may lead to higher morbidity and mortality, mainly linked to cardio-vascular disorders (such as heart attacks and strokes). Thus, control of blood pressure should be a goal to be pursued by all health professionals.

It is important to mention that hormones play an important role in hypertension. The renin-angiotensinaldosterone axis controls the sodium and potassium balance and arterial blood pressure. The atrial hormone is also reported to counter-influence the renin-angiotensin-aldosterone axis in situations involving high blood pressure or sodium surfeit ${ }^{18}$. However, more studies are required to further elucidate the hypertension etiopathogenesis. Heart rate is expressed as the number of heart beats per minute. Problems with heart rhythm and rate are quite common in the elderly. Low heart rate (bradycardia) and arrhythmias such as atrial fibrillation are also common. The results of this study showed an average of $71.4 \mathrm{bpm}$, with maximum and lowest values of 100 and 43 , respectively. The results of this study are in agreement with those of previous studies, ranging from 60 to $100 \mathrm{bpm}$ at rest $^{9}$ and an average of $69.6 \mathrm{bpm}^{17}$. No correlation was observed between blood pressure and AUDIT scores, which differs from a classic study ${ }^{19}$ that evaluated the influence of alcohol consumption and blood pressure on 83,947 adult patients and found that regular use of three or more drinks of alcohol per day should be considered a risk factor for hypertension due to its increase in systolic and diastolic pressures as well as higher prevalence of pressures higher than $160 / 95 \mathrm{mmHg}$. This discrepancy between the results presented here and those from Klatsky et al. ${ }^{19}$ is probably due to the smaller sample size in the present study, as the number of participants showing characteristics of alcohol addiction was very small, weakening the statistical power to detect a possible correlation between blood pressure and AUDIT scores.

The concepts proposed by the American Diabetes Association were used to classify the obtained levels of blood glucose. Two hours after meals, normal blood glucose should be less than $140 \mathrm{mg} / \mathrm{dL}^{11}$, and higher values are indicative of diabetes. Using these parameters, it was found that $80.2 \%$ of the subjects had levels within the normal range of blood glucose and $18.6 \%$ had levels indicative of diabetes. De Deco et al. ${ }^{9}$ found $32 \%$ of diabetes among institutionalized elderly and 30\% among community-dwelling elderly. The lower incidence of diabetes found in this study can be attributed to health programs targeted at the elderly, using both controlled diet and specific medication. Furthermore, no correlation was observed between blood glucose with 
AUDIT scores. As mentioned above, the number of participants showing characteristics of alcohol addiction in the present study was very small, weakening the statistical power to detect a possible correlation between blood glucose and AUDIT scores. Further studies should also test the possible influence of eating habits and even oral health, which interferes on the chewing pattern, on diabetes prevalence.

The high prevalence of hypertension in this study, despite the high use of antihypertensive drugs is probably due to the low adhesion of patients to the anti-hypertensive treatment (patients do not take their medications as they should and/ or do not honor recall appointments as they should). Thus the observed hypertension levels were high, despite the patients' claim to use the anti-hypertensive medicines. It should signal red to the dentist when the patient tells that he/she has controlled hypertension. The dentists do better double-checking though. Regarding the diabetes, prevalence and use of medicines were low in this group, which indicates a greater participation of this group of elderly individuals in the treatment of diabetes. It may be due to obesity control and personal appearance, which is increasingly prized by aged people, especially in this group, which leads a fairly active social life.

The most used drugs by the elderly were the ones to control hypertension $(37.48 \%)$, cholesterol $(9.54 \%)$, hormone replacement therapy or hormone supplementation in patients with hypothyroidism $(7.75 \%)$, and hypoglycemic drugs $(6.04 \%)$, with an average of 2.2 medications used daily. Similar results were found by De Deco et al. ${ }^{9}$, who found an average of 1.78 medicines for non-institutionalized elderly. In other countries, such as Finland, it was verified that elders use more drugs on a daily basis $(7.9 \text { drugs })^{20}$ than they do in Brazil. The relatively low use of drugs among the elderly population should be seen as a positive fact, since a good control of health parameters is achieved, because it reduces the risk of iatrogenic effects of drugs and drug interactions. However, it may also be caused by the low income of the elderly in Brazil, because cost plays an important role in the choice of drugs ${ }^{8}$. Some of the most used drugs in the present study, such as simvastatin, captopril and insulin, have government subsidies and are purchased by low-income populations at almost $10 \%$ of the normal over the counter cost, since they are considered as essential drugs by the Brazilian Ministry of Health ${ }^{8}$.

The relatively low use of drugs among this elderly population may also be related with the absence of regular recall examinations, which may lead to a lower rate of diagnoses, leading to a lower use of drugs. The absence of regular recall examinations is a chronic problem in the Brazilian health system, as access to physicians is not universal.

It is important to consider that a low medication intake may affect the control of several health parameters, which may negatively influence the general health of the patients. However, a limitation of this study is related to the absence of drug dosage verification, and without dosage records it is not possible to check for problems related to this variable. It is also important to consider that the low medication intake may affect the control of several health parameters, which may negatively influence the general health of the patients. Women presented an average use of 2.3 medicines on a daily basis, while men used 1.7. These results were expected, because women visit medical clinics and seek to prevent diseases more often than men, showing more concern about health, while men mostly seek medical advice only when they are already experiencing a health problem.

The AUDIT allowed evaluating the use of alcohol in this elderly sample. It can be argued that specific tests such as AUDIT have a better performance in the elderly compared to young people ${ }^{21}$, and AUDIT seems to be a reliable method to identify alcohol-related problems and assist in planning appropriate services for the treatment and prevention of alcoholism ${ }^{4}$. To calculate the frequency of alcohol intake, scores were assigned to each question, and after that the final score was obtained. Questions 1 to 8 covered aspects such as quantity, frequency and the way the person usually ingest alcohol. The answer scores ranged from 0 to 4 points and the answers to questions 9 ("Have you ever got hurt or hurt someone because you have drunk?") and 10 ("Anytime a relative, friend, doctor, or health care professional expressed concern for your alcohol intake or suggested that you should quit drinking?") are answered with 0,2 , or 4 points. The results are expressed in values between 0 and 40. Scores ranging from 1 to 7 are considered low-level drinkers; from 8 to 19 are considered harmful abuse and scores ranging from 20-40 are considered alcohol addiction.

The present results showed a higher prevalence of lowlevel intake of alcohol (91.0\%), 7.8\% for harmful use / abuse, and only $1.0 \%$ of dependency. These results are similar to those of Jeong et al. ${ }^{22}$, who conducted a study involving 997 elderly Koreans and observed that only $23 \%$ of the sample consumed alcohol. Among those who consumed alcohol, 150 met the criteria for social drinking, 46 for atrisk drinking, 17 for alcohol abuse, and 23 for alcohol dependence at the baseline assessment ${ }^{22}$.

In a recent study ${ }^{23}$, it was verified that only $23.6 \%$ in a sample of 169 elderly men from the metropolitan region of São Paulo were lifetime abstainers and $38 \%$ could be considered alcohol addicts in the past. However, the results of such a study cannot be directly compared to the results of the present study because these authors evaluated alcohol consumption in the past, and the present study used a specific tool to measure the current alcohol intake (AUDIT).

The predominance of women in the present sample may also have influenced the results regarding alcohol intake. In this sample, comparing alcohol consumption among sexes women showed lower intake of alcohol compared to men. These results are in agreement with Kim et al. ${ }^{24}$, in which alcohol was reported as a common problem especially among men. A previous study by Denneson et al. ${ }^{12}$ evaluated, by a cross-sectional study, a sample of 1,105 subjects aged 90 years in which $96.19 \%$ were men that took part in World War II. The alcohol intake measurement was also made by 
AUDIT and their results showed that $60.3 \%$ of the men were abstainers, $36.9 \%$ were low-level drinkers, and only $2.8 \%$ were medium- or high-level drinkers, while $46.9 \%$ of the women were abstainers, $43.6 \%$ were low-level drinkers, and the remaining $9.5 \%$ were medium-level drinkers. The comparison of these results with the findings of the present study should take into consideration that the sample in this study was mostly composed by women and the average age was 69.5 years old, which differs from the above-mentioned study $^{12}$. Another study ${ }^{22}$ also affirmed that no women reported high-level alcohol consumption.

When the use of medicines and alcohol intake were evaluated, it was possible to verify that patients who took a higher number of drugs made lower use of alcohol, decreasing the risk of alcohol-drug interactions. Patients older than 65 years have a significantly increased risk for drug-related problems not only because aging affects how their body handles medications but also because they take more drugs when compared with younger patients ${ }^{8}$. Adverse side effects in older patients are a common cause for hospitalization and an important cause of morbidity and death ${ }^{25}$. Side effects are associated with polypharmacy. Carbonin et al. ${ }^{26}$ suggested that the risk of adverse side effects is rather exponentially than linearly related to the number of medicines taken by the patient. Also, the adverse side effects lead to increased expenses for the public health system. The Audit Commission $^{27}$ found that each year the NHS (UK) spends approximately $£ 0.5$ billion for longer stays in hospitals due to errors in medicines and medication. As raised by the present study hypothesis, the concomitant use of drugs and alcohol by the elderly may decrease the efficacy of the drugs and increase the incidence of undesirable side effects ${ }^{7,9}$.

In this study, it was possible to verify high prevalence of hypertension, a relatively low incidence of diabetes, and low use of drugs, where antihypertensives and antilipidemics were the most used, since they are available for free under governmental assistance ${ }^{8}$. Women use more daily medications than men. Low alcohol consumption was also observed, and women consume less alcohol than men. The generalization of the results presented here should be made with caution, since a specific group with some homogeneous variables, such as social status and access to full health services, may not be the reality of the entire population of this city.

\section{Acknowledgments}

This study was presented as an oral presentation in the 36th Annual Conference of the European Prosthodontic Association, in Rotterdam. This study received financial support from a research agency (FAPESP 2010/16121-3).

\section{References}

1. Brazilian Institute of Geography and Statistics. National Survey by Household Sample. Brasília: IBGE; 2008.

2. Sorocco KH, Ferrell SW. Alcohol use among older adults. J Gen Psychol. 2006; 133: 453-67.
3. Culberson JW. Alcohol use in the elderly: beyond the CAGE. Part 1 of 2 prevalence and patterns of problem drinking. Geriatrics. 2006; 61:23-7.

4. Philpot M, Pearson N, Petratou V, Dayanandan R, Silverman M, Marshall J. Screening for problem drinking in older people referred to a mental health service: a comparison of CAGE and AUDIT. Aging Ment Health. 2003; 7:171-5.

5. Suwala M, Gerstenkorn A. Detection of alcohol problems among elderly people. Psychiatr Pol. 2007; 41: 703-13.

6. Sedlak CA, Doheny MO, Estok PJ, Zeller RA. Alcohol use in women 65 years of age and older. Health Care Women Int. 2000; 21: 567-81.

7. Moore AA, Beck JC, Babor TF, Hays RD, Reuben DB. Beyond alcoholism: identifying older, at-risk drinkers in primary care. J Stud Alcohol. 2002; 63: 316-24.

8. Marchini AMPS, de Deco CP, Silva MRV, Lodi KB, Rocha RF, Marchini L. Use of medicines among a Brazilian elderly sample: a cross-sectional study. Int J Gerontol. 2011; 5: 94-7.

9. de Deco CP, do Santos JF, da Cunha V de P, Marchini L. General health of elderly institutionalised and community-dwelling Brazilians. Gerodontology. 2007; 24: 136-42.

10. Santos G, Barreto E, Santos JF, Marchini L. Alcohol and quality of life among social groups for the elderly in São José dos Campos, Brazil. Appl Res Qual Life 2013: 1-9. doi: 10.1007/s11482-013-9225-y.

11. American Diabetes Association. ADA live from American Diabetes Association [internet]. 2011 [accessed 2011 May 2]. Available from: http://www.diabetes.org.

12. Denneson LM, Lasarev MR, Dickinson KC, Dobscha SK. Alcohol consumption and health status in very old veterans. J Geriatr Psychiatry Neurol. 2011; 24: 39-43.

13. Saunders JB, Aasland OG, Babor TF, de la Fuente JR, Grant M. Development of the alcohol use disorders identification Test (AUDIT): WHO Collaborative Project on Early Detection of Persons with Harmful Alcohol Consumption-II. Addiction. 1993; 88: 791-804.

14. Méndez EB. A Brazilian version of AUDIT - Alcohol use disorders identification test [mastering thesis]. Pelotas: Federal University of Pelotas; 1999.

15. Blood Pressure UK. Blood pressure chart [internet]. 2008 [accessed 2014 Jan 4]. Available from: http://www.bloodpressureuk.org/ BloodPressureandyou/Thebasics/Bloodpressurechart.

16. Zeitune MP, Barros MB, Cesar CL, Carandina L, Goldbaum M. Arterial hypertension in the elderly: prevalence, associated factors and control practices in Campinas, São Paulo, Brazil. Cad Saude Publica. 2006; 22: 285-94.

17. Matsumura K, Ansai T, Awano S, Takehara T, Abe I, lida M, et al. Association of dental status with blood pressure and heart rate in 80-yearold Japanese subjects. Jpn Heart J. 2003; 44: 943-51.

18. Laragh $\mathrm{JH}$. Atrial natriuretic hormone, the renin-aldosterone axis, and blood pressure-electrolyte homeostasis. N Engl J Med. 1985; 313: 133040.

19. Klatsky AL, Friedman GD, Siegelaub AB, Gerard MJ. Alcohol consumption and blood pressure Kaiser-Permanente Multiphasic Health Examination data. N Engl J Med. 1977; 296: 1194-200.

20. Hosia-Randell HM, Muurinen SM, Pitkala KH. Exposure to potentially inappropriate drugs and drug-drug interactions in elderly nursing home residents in Helsinki, Finland: a cross-sectional study. Drugs Aging. 2008; 25: 683-92.

21. Berks J, McCormick R. Screening for alcohol misuse in elderly primary care patients: a systematic literature review. Int Psychogeriatr. 2008; 20: 1090-103.

22. Jeong HG, Kim TH, Lee JJ, Lee SB, Park JH, Huh Y, et al. Impact of alcohol use on mortality in the elderly: results from the Korean Longitudinal Study on Health and Aging. Drug Alcohol Depend. 2012; 121: 133-9.

23. de Oliveira JB, Santos JL, Kerr-Correa F, Simao MO, Lima MC. Alcohol screening instruments in elderly male: a population-based survey in metropolitan Sao Paulo, Brazil. Rev Bras Psiquiatr. 2011; 33: $347-52$. 
24. Kim KW, Choi EA, Lee SB, Park JH, Lee JJ, Huh Y, et al. Prevalence and neuropsychiatric comorbidities of alcohol use disorders in an elderly Korean population. Int J Geriatr Psychiatry. 2009; 24: 1420-8.

25. Routledge PA, O'Mahony MS, Woodhouse KW. Adverse drug reactions in elderly patients. Br J Clin Pharmacol. 2004; 57: 121-6.

26. Carbonin P, Pahor M, Bernabei R, Sgadari A. Is age an independent risk factor of adverse drug reactions in hospitalized medical patients? J Am Geriatr Soc. 1991; 39: 1093-9.

27. Anon A. A spoonful of sugar; medicines management in NHS hospitals. London: Audit Commission; 2001. 